\title{
ANTIMICROBIAL PROPHYLAXIS AND STEWARDSHIP IN NURSING HOME: A NARRATIVE REVIEW
}

\author{
M. TANDAN \\ Corresponding author: Meera Tandan, PhD, Cecil G Sheps Centers for Health Service Research, University of North Carolina, USA, Email -meerat@email.unc.edu, \\ ORCID = orcid.org/0000-0002-7742-7980
}

\begin{abstract}
The rapid increase in antimicrobial resistance is a great concern in safeguarding the nursing home population. Improving inappropriate prescribing is the main agenda of antimicrobial stewardship. It is complicated to articulate the appropriateness of antimicrobial prescribed in nursing home residents, especially the prophylaxis. It is tricky because most of the residents are often on multiple medications, and there are no guidelines available for prescribers to decide on the appropriateness. Except for urinary tract infections, prescribing instructions rarely exists for other infections in nursing homes. Very few studies discuss prophylactic prescribing, and most of them are prevalence studies. These studies showed the urinary tract, respiratory tract, and skin-related problem as the most common cause of antimicrobial prophylaxis. However, this information is presented as total proportion without disaggregated analysis of conditions and types of the urinary and respiratory tract and skin-related infections. Further, the definition used is vague, and significant risk factors of antimicrobial prophylaxis and precision about the use remain unanswered. Lack of appropriate definition and unanswered questions are potentially challenging for stewardship in nursing homes. This review summarizes the current situation of antimicrobial prophylaxis and discusses gaps in the literature and pertinent areas for moving forward for antimicrobial stewardship in nursing home settings.
\end{abstract}

Key words: Prophylactic, antimicrobials, review, stewardship, nursing homes, urinary tract infection, respiratory tract infection, skin infections.

\section{Introduction}

Antimicrobial resistance (AMR) is a global issue. In the United States (US), more than 2.8 million antimicrobialresistant infections occur each year, and above 35,000 people die due to AMR (1). One of the critical causes of AMR is inappropriate prescribing. It could be either prescribing for no reasons or preventing infection, choosing wrong antimicrobials or prescribing correct antimicrobials but incorrect dose or duration, or prescribing against the guidelines $(2,3)$. None can avoid antimicrobials and the risk of getting resistant bacteria in their lifetime. Though, the older people living in nursing homes (NHs-also known as long-term care facilities) are more likely to get infections and AMR (4). Every year, 1.6 to 3 million infections occur in NHs and between $40-75 \%$ of the prescribed antimicrobials are inappropriate (5). In the US, $11 \%$ of $\mathrm{NH}$ residents receive antimicrobials in a single day. Nearly $40 \%$ lack prescription information (6). Similarly, 9\% antimicrobials are prescribed in Australian NHs, of which $40 \%$ do not meet bacterial infection criteria (7). In Europe, antimicrobial use in $\mathrm{NH}$ varies across countries (range 1-12\%), and $27 \%$ are prophylaxis (8).

Antimicrobial prophylaxis (AP) is an effective intervention in preventing infections; yet, it has become an inappropriate practice in NH. For example, $80 \%$ of prophylactic antimicrobials are without termination or end date, and many of them are broad-spectrum in nature, such as fluoroquinolones $(2,9)$. Such prescribing behavior indicates that AP becomes an essential reason for irrational prescribing and might be the leading cause of AMR in NH. Addressing prophylactic prescribing is central to attain the motto of antimicrobial stewardship. While much attention has not been given to this area, and very few literatures published. This study aims to provide the extent to which antimicrobials are prescribed prophylactically, including challenges in attaining stewardship in NH settings.

\section{Prophylactic Antimicrobial Prescribing}

The term prophylaxis means the prevention of infections. Prophylaxis is not only limited to preventing disease occurrence, but it can also prevent the disease's worsening and minimize the severity. For example, preoperative prophylaxis used to avoid initial infection as well as to reduce the severity. Sometimes, prophylaxis measures are applied to prevent recurrence or reactivation of diseases (e.g., prevention of recurrent urinary tract infections (UTI) (5).

AP varies across countries and regions $(8,10)$. In NHs, overall prevalence lies between $4-53 \%$ in European countries (8), 26\% in Australia (7), and 23\% in the US (6). In some countries such as Denmark, Finland, Norway, and UK-Northern Ireland, prophylactic use is higher than the therapeutic (10). A study in Michigan reported that $17 \%$ of the antimicrobial prescriptions were without clinical or laboratory evidence of infections (11), potentially the indication was for prevention. Antimicrobials are often prescribed to prevent most common infections -mainly related to urinary and respiratory tract and skin. A comparative study in Irish NHs reported $70-75 \%$ of AP for urinary tract, 16-26\% for respiratory tract and $8-9 \%$ for skin or wound-related conditions in $\mathrm{NH}$ 
(9). Likewise, a similar trend reported from other European countries $(10,12)$ and Australia (13).

\section{Prophylaxis related to urinary tract}

A vital issue in the management of UTI in NH is inappropriate antimicrobial use, where prophylaxis accounted for $22 \%$ of antimicrobials prescribed (8). The prevalence is above $30 \%$ in Belgium, Denmark, Finland, Ireland, Norway, Sweden, and the UK (8), and $25 \%$ in the US (14). Prescribing in $\mathrm{NH}$ resident are misguided by the positive urine culture results and mild pyuria, and are almost treated with antimicrobials. As asymptomatic bacteriuria increases with age and practically all $\mathrm{NH}$ residents (asymptomatic) tests positive bacteriuria (15). Antimicrobial either therapeutic or prophylactic for asymptomatic bacteriuria is not recommended (16). A previous study demonstrated no benefits of antimicrobials in reducing symptomatic UTI (17). Still, there is a practice of antimicrobial prescribing for such conditions. But, the exact prophylactic contribution of asymptomatic bacteria is unknown, an auspicious area for exploration.

A urinary catheter is another cause of AP. About 7-9\% of the residents in NH had a urinary catheter $(18,19)$. Urinary catheter increases the risk of asymptomatic bacteriuria, subsequently increase antimicrobial use as described above. UTI guidelines do not emphasize AP for a resident with a urinary catheter (20). Nevertheless, a urinary catheter viewed as a straightforward indication for antimicrobial prescribing and, more often, prophylaxis in the NH. A study reported that a resident with a urinary catheter is nearly two times more likely to be on AP than those without catheter (9). A Cochrane review indicated weak evidence of AP in reducing bacteriuria (symptomatic and symptomatic) in adult patients with a urinary catheter. However, there remains a higher heterogeneity among the included studies (21). Moreover, few studies describe the use of prophylactic antimicrobials during insertion and removal of a catheter for UTI (22). But, the outcome of the study describe was not strong enough to support prophylaxis and did not include the patients from NH. Additionally, guidelines from the US and Europe do not advocate AP for either of the cases or the patients undergoing urological procedure unless it is essential because of AMR issues $(20,23,24)$.

Recurrent UTI (at least three UTI per year or two in the last six months) is another apparent reason for AP. In older people, both UTI or recurrent UTI are over diagnosed and over treated (25). In NHs, $12-14 \%$ of older adults with recurrent UTI receive AP.26 Guidelines recommendation for recurrent UTI prophylaxis varies-- American Urological Association moderately recommends UTI prophylaxis to those with recurrent UTI and emphasize that prescriber should always outweigh benefit than the risk (27). While the UK guidelines commend a daily use of low dose prophylaxis for recurrent UTI if initial single-dose prophylaxis fails to prevent UTI's re-occurrence (28). Nevertheless, these guidelines are silent about recurrent UTI prophylaxis in NH residents about when to start and for how long. A study of a large cohort of older people in UK indicated that prophylaxis for recurrent UTI significantly reduces the risk of clinical recurrence of UTI and UTI related hospitalization in men (26). The study has the limitation of exclusion of microbiological data in the analysis. Hence, it is unclear if the AP genuinely meets the definition of recurrent UTI. The current increasing trend of AMR and adverse events associated with antimicrobials suggests further investigation of the usefulness and practicality of recurrent UTI prophylaxis in NHs.

The common prophylactic antimicrobials include trimethoprim $(30 \%)$, nitrofurantoin $(27 \%)$, methenamine $(12 \%)$, cefalexin $(6 \%)$, and fosfomycin $(6 \%)$ for urinary infections. These antimicrobials cover $81 \%$ of all prophylactic antimicrobials prescribed in NHs (8). The proportion of use and antimicrobials types differ between countries. For example, nitrofurantoin and trimethoprim more often used in Irish NHs (9), and methenamine in Norwegian (29). These are the approved first-line treatment option for UTI. Moreover, for recurrent UTI, the preventive use of these antimicrobials did not show significant clinical and microbiological cure rates in adult women (30). Consequently, the use of these antimicrobials is associated with increased risk of bacterial resistance and side effects, encouraging for limited use in older people. Trimethoprim is frequently associated with a higher risk of acute kidney injury and hyperkalemia (31), and its resistance to uropathogens is above $35 \%$ in $\mathrm{NH}$ population $(32,33)$. In contrast, nitrofurantoin did not show resistance to uropathogens (33) and has proven useful for acute and severe UTI, including the catheter-associated if prescribed prophylactically (either shorter or longer duration) (34). Meanwhile, nitrofurantoin cause pulmonary fibrosis, hepatoxicity, and symptoms specific to gastrointestinal in older adults $(30,34)$. So far, methenamine prophylaxis has proven effective in reducing symptomatic UTI in older adults without resistance to uropathogens and low adverse events (35). Its use is sometimes problematic in residents without adequate renal function and with renal tract abnormalities, and these conditions are rarely not present in $\mathrm{NH}$ residents. Therefore, in total, the AP, if necessary, should be based on clinical judgment balancing the risk and benefit without excluding the local resistance profile of antimicrobials prescribed.

\section{Prophylaxis related to respiratory tract}

Overall, prophylaxis related to respiratory tract shares $6 \%$ of the total antimicrobials used in NHs (8). The highest prescribed in Netherland (27\%), Portugal (23\%), Italy (9\%), Ireland (9\%), Sweden $(8 \%)$, and UK $(6 \%)(8,36)$. These prophylactic prescribing includes both the infection of the upper and lower respiratory tract (10). A separate analysis, specific to different types of respiratory infection, is not reported. In the US, AP is $38 \%$ for aspiration pneumonia (37). In sum, the prescription for acute bronchitis could be a more prominent contributor to prophylaxis related to respiratory infections 


\section{THE JOURNAL OF NURSING HOME RESEARCH SCIENCESC}

in NHs. Most of the acute bronchitis is viral in etiology. Overprescribing more often connected to acute bronchitis, compromising $40 \%$ of all prescriptions (38). Guidelines do not suggest antimicrobials for acute bronchitis, except confirmed by a diagnostic test or suspected for pertussis or unless the risk of a severe complication due to comorbidity (39). Also, no clinical benefits of antimicrobials in the treatment of acute bronchitis reported for patients above 60 years. Alternatively, AP may have suggested in acute cough for patients with a history of hospitalization, diabetes mellitus, congestive heart failure, or patients on steroids; but, not for long-term use in stable patients.

The other reason of AP for respiratory problems could be chronic obstructive pulmonary disease (COPD) and pneumonia. It is mainly because $20 \%$ of hospitalizations of $\mathrm{NH}$ residents are from these infections where most of the antimicrobials initiated (40). A study demonstrated that AP substantially reduces the number of exacerbations and improves the quality of life of older people with COPD (41). Moreover, the routine AP is not suggested for acute exacerbation of COPD as the prophylaxis duration is quite lengthy (12 to 36 months) (39). Concerning pneumonia, AP significantly reduces the incidence and mortality in the intensive care unit (42), but no clinical benefits for older people in outpatient settings (37). The finding implies benefits of AP to some extent. Nevertheless, long term use for more than a year in older adults in outpatients subsequently escalate frequent use leaving no antimicrobial free days. Such a situation is more likely to provide a conducive environment for AMR, hence these prescribing issues with $\mathrm{NH}$ required further research attention.

For respiratory infections, macrolides, cephalosporin, and tetracycline are the conventional AP.8 Of the total prophylaxis, macrolides represent 47-69\%, tetracycline 11-16\% (most importantly, the doxycycline), and cephalosporin 3-11\%.9 Macrolides prophylactics could be associated with COPD, as $27 \%$ of $\mathrm{NH}$ residents had COPD.40 In this study, the prophylactic use of these antimicrobials is not subject to either COPD or pneumonia. Macrolide causes adverse drug events if used long-term in patients under medication that prolonged QT interval. For example, an antipsychotic medication. Nearly a quarter of the $\mathrm{NH}$ residents receive one or more antipsychotic drugs (43). Therefore, understanding the appropriateness of prophylactic antimicrobials in this population is imperative. Further, desegregation analysis of AP by types of respiratory problems and appropriateness of prophylaxis merits advantages in reducing adverse drug events and AMR in NH residents.

\section{Prophylaxis related to skin problem}

Skin infection is the third most common problem, accounting for $20 \%$ of all infections in NH (8). AP for skinrelated problems ranges from $10-12 \%(9,36)$. The skin infection includes cellulitis, soft tissue, and wound infection, while a discrete analysis of AP is not available for specific skin conditions. In $\mathrm{NH}$, cellulitis (mostly non-purulent) is a common skin problem often confused with non-bacterial chronic inflammation, dermatitis occurring in the lower extremities (38). Therefore, these problems are misdiagnosed, misinterpreted in many residents, and subsequently mistreated and prescribed inappropriately, which may include AP. Of the total misdiagnosed cellulitis cases, $92 \%$ receive inappropriate antimicrobials (44). AP is moderately recommended for recurrent cellulitis (3-4 episodes per year) for 4-52 weeks (45); however, no clear indication exists specific to $\mathrm{NH}$ resident. As AP may reduce future recurrent cellulitis, prescribing for a longer duration may likely to develop AMR and associated adverse events. Therefore, a detailed study on skin-related AP in $\mathrm{NH}$ is vital to curb the increase AMR issue and accomplish stewardship. Similarly, due to a lack of standardized guidelines, there is a significant dilemma in both diagnosis and antimicrobial use for pressure ulcers and abscess. Meanwhile, antimicrobials have shown no clinical benefit, and prophylaxis is not suggestive for wound infection (45).

On a similar note, studies on types of antimicrobials (either therapeutic or prophylactic) used for skin infections for $\mathrm{NH}$ are very less. In the US, oral beta-lactam (cephalexin, amoxicillin/ clavulanate), tetracycline (doxycycline), and fluoroquinolones are common therapeutics for skin infections (46). Whilst, this study did not discuss prophylactic. An Irish study showed that of the skin-related AP, 65-68\% are tetracycline, 8-26\% penicillin, and 3-6\% macrolides in NH (9). Tetracycline, particularly doxycycline, was reported from intellectual disability facilities. These are the facilities where residents are young, and prolonged use of doxycycline is an advised option for acne vulgaris in Ireland. Every country has its definition of NH and guidelines for antimicrobials, so the prescribed antimicrobials' local AMR profile should be considered. At the same time, further detailed exploration is required to understand the issue and suppress the unwanted skin-related AP problems in $\mathrm{NH}$.

\section{Literature-related to Antimicrobial Prophylaxis}

Very few pieces of literature are available on AP in $\mathrm{NH}$ (Figure 1). With the introduction of a stewardship concept in the infection control in $\mathrm{NH}$, the number of scientific studies substantially increased for antimicrobial stewardship. But then, the literature on AP is scarce. There are 41 published papers available till May 27, 2020, in the PubMed database. The main objective of most of the published papers was not prophylactic prescribing. Of the 41 studies, 18 studies from Europe, 15 the US, 4 UK, and 2 Canada. About $25 \%$ of the studies are related to the microbiological aspect, but with a little information on AP. If included, AP either mentioned in the discussion or conclusion sections. The majority of the European studies are related to AP for any cause/infections (11 studies) and UTI (4 studies), while from the US are related to UTI (6 studies) and pneumonia and Invasive Group A Streptococcal 


\section{ANTIMICROBIAL PROPHYLAXIS AND STEWARDSHIP IN NURSING HOME}

Disease (iGAS-4 studies) (Figure 2). Compared to the US, studies conducted in Europe are explicit about AP, but the data presented are just limited to prevalence. The higher number of studies in Europe is the effect of a few AP data collected as a part of point prevalence surveys. In Europe surveillance study on healthcare-associated infections in long-term care facilities (HALT) began in 2008 and has already completed three consecutive studies.8,10 The definition used for collecting AP data was vague and differed across countries. The analysis was mainly presented descriptively as a proportion. Except for a study published in 2019 in Eurosurveillance, 9 none of the studies had performed an in-depth analysis of prophylactic prescribing. This study compares the result of two surveys and identified the risk factors of AP in NH.

\section{Figure1}

Growth of literature related to antimicrobials use, prophylactic antimicrobial use, and antimicrobial stewardship in NHs or in LTCFs (till May 27, 2020)

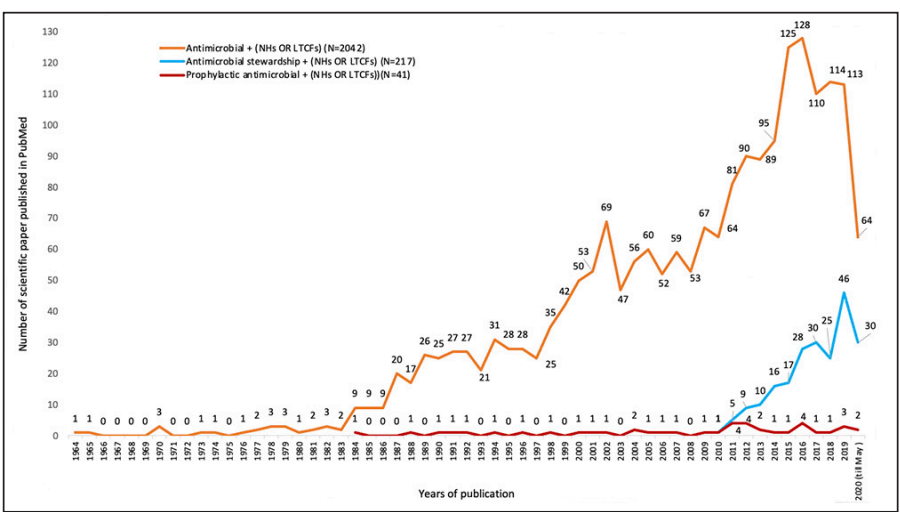

Figure 2

Prophylactic papers and the disease conditions studied in the literature by countries

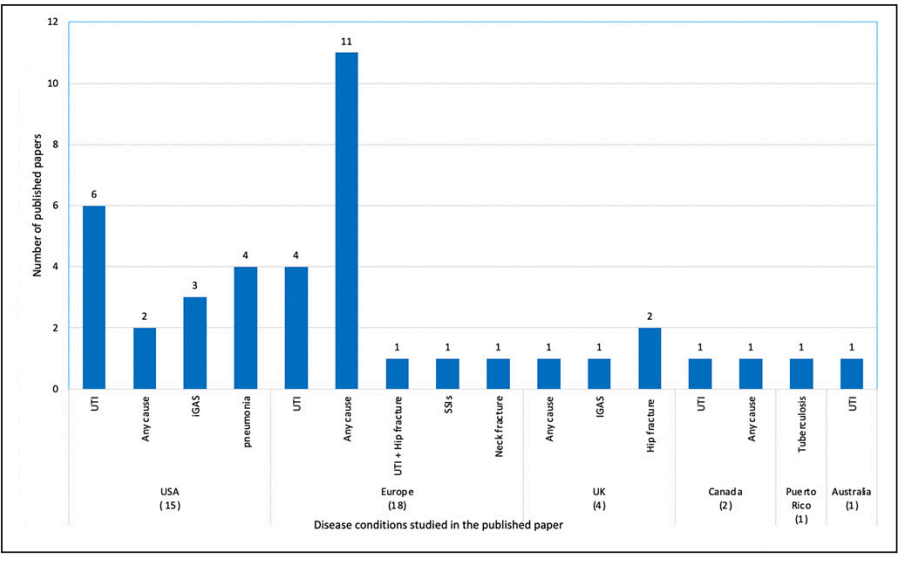

Similarly, in 2009, the Center for Disease Prevention and Control (CDC) launched a pilot point prevalence survey in $9 \mathrm{NH}$ in the 4 Emerging Infection Program (EIP) states and collected information on prophylactic antimicrobial prescribing (47). A paper from this pilot study mostly focuses on UTI prophylaxis.48 Following the pilot study, a full-scale survey was conducted in $161 \mathrm{NHs}$ in 2017 in the 10 EIP states. Recently, a paper has been published as an outcome of this study; however, is UTI centered.49

Besides, studies from Canada were a prevalence 50 and a review on AP (51). The review study included a little argument on the prophylactic prescribing and indwelling urethral catheter in NH residents.51 A study in Puerto Rico discussed antimicrobials' use, isoniazid as a preventive therapy for tuberculin positive test for the prevention of active tuberculosis in the $\mathrm{NH}$ residents (52).

\section{Antimicrobial Prophylaxis and Stewardship}

Antimicrobial stewardship is directly proportional to rational prescribing, meaning prescribed only when required in appropriate quantity and duration. In the current situation of AMR tragedy, antimicrobial stewardship practice is critical in NH settings. A study showed that antimicrobial stewardship practices reduce the prevalence of antimicrobial consumption by $20 \%$ in $\mathrm{NH}(18)$. In the US, all NH required to implement antimicrobial stewardship programs since 2017 (33). According to a recent report, $33 \%$ of $\mathrm{NH}$ had a comprehensive antimicrobial stewardship policy (53). Additionally, antimicrobial stewardship guidelines do not explicitly discuss prophylactic prescribing in $\mathrm{NH}$, except asymptomatic bacteriuria (54). Considerable insight has been developed regarding inappropriate antimicrobial prescribing for successful stewardship in $\mathrm{NH}(14,21,30)$. The prophylactic prescribing remains broad and neglected. Guidelines specifically addressing the antimicrobial prescribing for $\mathrm{NH}$ are few, and most of them are silent about the prophylactic prescribing in particular that elucidate duration, and indications $(16,45,55$, 56). If mentioned, they are developed based on little evidence and have little association with definite practice and outcome. UTI is highly focused, but stewardship cannot be attained by only focusing on AP related to UTI only because RTI and skin infections are equally important infections contributing in the development of AMR in NH.

Likewise, $\mathrm{NH}$ residents are older people with long-term physical and cognitive impairments, chronic illness, multiple morbidities, and often exposed to indwelling devices and invasive procedures (5). These multiple and complicated problems could be the reasons providers baffle decision making and identify prophylactic prescribing as a natural option or to be on the safe side. Antimicrobials use directly linked to AMR, and studies have shown that antimicrobial stewardship can reduce antimicrobial prescribing and resistance $(33,57)$. Therefore, developing further understanding of AP should be an essential agenda for effective antimicrobial stewardship in $\mathrm{NH}$. The scope of AP use includes identifying the cause and appropriateness of prophylaxis and their amendment in the $\mathrm{NH}$ guidelines to improve prescribing and subsequently reduce the AMR. 


\section{THE JOURNAL OF NURSING HOME RESEARCH SCIENCESC}

\section{Conclusion}

Antimicrobial prophylaxis is a critical prescribing issue in NHs. More than two-thirds of the total prophylaxis are urinary tract-related, one-fourth respiratory tract, and one in ten skin-related problems. In the current literature, factors of UTI prophylaxis are unexplored except recurrent UTI. Similarly, there is a lack of disaggregated data on AP precisely by respiratory and skin problems for NHs. Guidelines are not clear about when and under which condition to prescribe or not to prescribe for common infections in NHs, specifically AP. Likewise, evidence supporting how appropriate AP practices in NHs is lacking, which necessitates future exploration of this area for effective antimicrobial stewardship implementation. In the absence of evidence of infection prevention and gravity limitation of prevalent infections, it is necessary to limit the prophylactic prescribing in NHs. Further studies are needed to clarify their efficacy and indication for appropriate antimicrobial stewardship in NHs.

Conflict of Interest: Author declare no conflict of interest.

Funding: None.

\section{References}

1. CDC. Antibiotic Resistance Threats in the United States.; 2019. https://www.cdc gov/drugresistance/pdf/threats-report/2019-ar-threats-report-508.pdf. Accessed July 5,2020 .

2. Tandan M, Burns K, Murphy H, Hennessy S, Cormican M, Vellinga A. Improving Antimicrobial Prescribing: A Multinomial Model Identifying Factors Associated With First- and Second-Line Prescribing. J Am Med Dir Assoc. 2019;20(5):624-628. doi:https://doi.org/10.1016/j.jamda.2018.10.028

3. CDC. Antibiotic Use in the United States, 2018 Update: Progress and Opportunities.; 2019. https://www.cdc.gov/antibiotic-use/stewardship-report/pdf/stewardshipreport-2018-508.pdf. Accessed October 4, 2020.

4. Montoya A, Cassone M, Mody L. Infections in Nursing Homes: Epidemiology and Prevention Programs. Clin Geriatr Med. 2016. doi:10.1016/j.cger.2016.02.004

5. Tandan M. Antimicrobial prescribing and stewardship in an ageing population. 2019 file:///C:/Users/meerat/Downloads/Thesis_Final_24th June 2019 Meera Tandan.pdf.

6. Thompson ND, LaPlace L, Epstein L, et al. Prevalence of Antimicrobial Use and Opportunities to Improve Prescribing Practices in U.S. Nursing Homes. In: Journal of the American Medical Directors Association. ; 2016. doi:10.1016/j. jamda.2016.08.013

7. Stuart RL, Wilson J, Bellaard-Smith E, et al. Antibiotic use and misuse in residential aged care facilities. Intern Med J. 2012. doi:10.1111/j.1445-5994.2012.02796.x

8. European Centre for Disease Prevention and Control. Point Prevalence Survey of Healthcare-Associated Infections and Antimicrobial Use in European Long-Term Care Facilities. April-May 2013. Stockholm.; 2014. doi:doi 10.2900/24172

9. Tandan M, O'connor R, Burns K, et al. A comparative analysis of prophylactic antimicrobial use in long-term care facilities in Ireland, 2013 and 2016. Eurosurveillance. 2019. doi:10.2807/1560-7917.ES.2019.24.11.1800102

10. Ricchizzi E, Latour K, Kärki T, et al. Antimicrobial use in european long-term care facilities: Results from the third point prevalence survey of healthcareassociated infections and antimicrobial use, 2016 to 2017. Eurosurveillance. 2018. doi:10.2807/1560-7917.ES.2018.23.46.1800394

11. Eke-Usim A, Gibson K, Rogers MAM, Mody L. Use of Diagnostic Testing Before Prescribing Antibiotics in Nursing Homes: Impact of Change in Function. Open Forum Infect Dis. 2015;2(suppl_1). doi:10.1093/ofid/ofv131.125

12. Rummukainen M-L, Kärki T, Kanerva M, Haapasaari M, Ollgren J, Lyytikäinen O. Antimicrobial prescribing in nursing homes in Finland: results of three point prevalence surveys. Infection. 2013;41(2):355-360. doi:10.1007/s15010-012-0331-9

13. Smith M, Atkins S, Worth L, Richards M, Bennett N. Infections and antimicrobial use in Australian residential aged care facilities: a comparison between local and international prevalence and practices. Aust Health Rev. 2013;37(4):529-534 doi:10.1071/AH12007

14. Thompson N, Penna A, Eure T, et al. Epidemiology of Antibiotic Use for Urinary Tract Infection in Nursing Home Residents. J Am Med Dir Assoc. 2019;21. doi:10.1016/j.jamda.2019.11.009

15. Nicolle LE. The paradigm shift to non-treatment of asymptomatic bacteriuria. Pathogens. 2016. doi:10.3390/pathogens5020038

16. Nicolle LE, Gupta K, Bradley SF, et al. Clinical practice guideline for the management of asymptomatic bacteriuria: 2019 update by the Infectious Diseases Society of America. Clin Infect Dis. 2019. doi:10.1093/cid/ciy1121

17. Nicolle LE. Asymptomatic bacteriuria: When to screen and when to treat. Infect Dis Clin North Am. 2003. doi:10.1016/S0891-5520(03)00008-4

18. Tandan M, Burns K, Murphy H, Hennessy S, Cormican M, Vellinga A Antimicrobial prescribing and infections in long-term care facilities (LTCF): a multilevel analysis of the HALT 2016 study, Ireland, 2017. Euro Surveill. 2018;23(46):1800278. doi:10.2807/1560-7917.ES.2018.23.46.1800278

19. McNulty C, Freeman E, Smith G, et al. Prevalence of urinary catheterization in UK nursing homes. J Hosp Infect. 2003;55(2):119-123. doi:10.1016/s01956701(03)00224-x

20. Hooton TM, Bradley SF, Cardenas DD, et al. Diagnosis, Prevention, and Treatment of Catheter-Associated Urinary Tract Infection in Adults: 2009 International Clinical Practice Guidelines from the Infectious Diseases Society of America. Clin Infect Dis. 2010;50(5):625-663. doi:10.1086/650482

21. Niël-Weise BS, van den Broek PJ, da Silva EM, Silva LA. Urinary catheter policies for long-term bladder drainage. Cochrane Database Syst Rev. 2012. doi:10.1002/14651858.cd004201.pub3

22. Lusardi G, Lipp A, Shaw C. Antibiotic prophylaxis for short $\square$ term catheter bladder drainage in adults. Cochrane Database Syst Rev. 2013;(7). doi:10.1002/14651858. CD005428.pub2

23. HSE. Guidelines for the Prevention of Catheter-Associated Urinary Tract Infection.; 2011. https://www.hpsc.ie/a-z/microbiologyantimicrobialresistance/ infectioncontrolandhai/guidelines/File,12913,en.pdf.

24. Tenke P, Kovacs B, Johansen T, Matsumoto T, Tambyah P, Naber K. European and Asian guidelines on management and prevention of catheter-associated urinary tract infections. Int J Antimicrob Agents. 2008;31 Suppl 1:S68-78. doi:10.1016/j ijantimicag.2007.07.033

25. McMurdo ME, Gillespie ND. Urinary tract infection in old age: over-diagnosed and over-treated. Age Ageing. 2000;29(4):297-298. doi:10.1093/ageing/29.4.297

26. Ahmed H, Farewell D, Jones HM, Francis NA, Paranjothy S, Butler CC. Antibiotic prophylaxis and clinical outcomes among older adults with recurrent urinary tract infection: Cohort study. Age Ageing. 2019. doi:10.1093/ageing/afy 146

27. American Urological Association. Recurrent uncomplicated urinary tract infections in women: AUA/CUA/SUFU Guideline (2019). American Urological Association. https://www .auanet.org/guidelines/recurrent-uti. Published 2019.

28. NICE. Urinary tract infection (recurrent): antimicrobial prescribing NICE guideline [NG112]. National Insitutue of Health Care Excellence ( UK). https://www.nice. org.uk/guidance/ng112/chapter/recommendations\#choice-of-antibiotic-prophylaxis. Published 2018

29. Alberg T, Holen O, Blix HS, Lindbaek M, Bentele H, Eriksen HM. Antibiotic use and infections in nursing homes. Tidsskr Nor Laegeforen. 2017;137(5):357-361. doi:10.4045/tidsskr.16.0621

30. Price JR, Guran LA, Gregory WT, McDonagh MS. Nitrofurantoin vs other prophylactic agents in reducing recurrent urinary tract infections in adult women: a systematic review and meta-analysis. Am J Obstet Gynecol. 2016;215(5):548-560. doi:10.1016/j.ajog.2016.07.040

31. Crellin E, Mansfield KE, Leyrat C, et al. Trimethoprim use for urinary tract infection and risk of adverse outcomes in older patients: cohort study. BMJ. 2018;360:k341. doi:10.1136/bmj.k341

32. Tandan M, Timilsina M, Cormican M, Vellinga A. Role of patient descriptors in predicting antimicrobial resistance in urinary tract infections using a decision tree approach: A retrospective cohort study. Int J Med Inform. 2019;127:127-133. doi:10.1016/j.ijmedinf.2019.04.020

33. Tandan M, Sloane $\mathrm{P}$, Ward K, et al. Antimicrobial resistance patterns of urine culture specimens from 27 nursing homes: Impact of a two-year antimicrobial stewardship intervention. Infect Control Hosp Epidemiol. 2019;40:1-7. doi:10.1017/ice.2019.108

34. Huttner A, Verhaegh EM, Harbarth S, Muller AE, Theuretzbacher U, Mouton JW. Nitrofurantoin revisited: a systematic review and meta-analysis of controlled trials. J Antimicrob Chemother. 2015;70(9):2456-2464. doi:10.1093/jac/dkv147

35. Chwa A, Kavanagh K, Linnebur SA, Fixen DR. Evaluation of methenamine for urinary tract infection prevention in older adults: a review of the evidence. Ther Adv drug Saf. 2019;10:2042098619876749. doi:10.1177/2042098619876749

36. Thornley T, Ashiru-Oredope D, Beech E, et al. Antimicrobial use in UK longterm care facilities: results of a point prevalence survey. J Antimicrob Chemother. 2019;74(7):2083-2090. doi:10.1093/jac/dkz135

37. Dragan V, Wei Y, Elligsen M, Kiss A, Walker SAN, Leis JA. Prophylactic Antimicrobial Therapy for Acute Aspiration Pneumonitis. Clin Infect Dis. 2018;67(4):513-518. doi:10.1093/cid/ciy120

38. Sloane PD, Tandan M, Ward K Zimmerman S. Antibiotic stewardship in long-term care settings. Weber DJ, Talbot TR. Mayhall's Hospital Epidemiology and Infection Prevention. 5th Edition; 2020.

39. NICE. Antimicrobial prescribing guidelines. NICE UK. https://www.nice.org. uk/about/what-we-do/our-programmes/nice-guidance/antimicrobial-prescribingguidelines. Published 2018.

40. Tandan M, Zimmerman S, Sloane PD, Ward K, Daniels LM, Wretman CJ. Which Nursing Home Residents With Pneumonia Are Managed On-Site and Which Are Hospitalized? Results from 2 Years' Surveillance in 14 US Homes. Journal of the American Medical Directors Association. 2020 Dec 1;21(12):1862-8.

41. Herath SC, Normansell R, Maisey S, Poole P. Prophylactic antibiotic therapy for chronic obstructive pulmonary disease (COPD). Cochrane database Syst Rev. 


\section{ANTIMICROBIAL PROPHYLAXIS AND STEWARDSHIP IN NURSING HOME}

2018;10(10):CD009764-CD009764. doi:10.1002/14651858.CD009764.pub3

42. Liberati A, D'Amico R, Pifferi S, Torri V, Brazzi L, Parmelli E. Antibiotic prophylaxis to reduce respiratory tract infections and mortality in adults receiving intensive care. Cochrane Database Syst Rev. 2009. doi:10.1002/14651858. CD000022.pub3

43. Briesacher BA, Tjia J, Field T, Peterson D, Gurwitz JH. Antipsychotic use among nursing home residents. JAMA. 2013;309(5):440-442. doi:10.1001/ jama.2012.211266

44. Weng QY, Raff AB, Cohen JM, et al. Costs and consequences associated with misdiagnosed lower extremity cellulitis. JAMA Dermatology. 2017. doi:10.1001/ jamadermatol.2016.3816

45. Stevens DL, Bisno AL, Chambers HF, et al. Practice Guidelines for the Diagnosis and Management of Skin and Soft Tissue Infections: 2014 Update by the Infectious Diseases Society of America. Clin Infect Dis. 2014;59(2):e10-e52. doi:10.1093/cid/ ciu296

46. Yogo N, Gahm G, Knepper BC, Burman WJ, Mehler PS, Jenkins TC. Clinical Characteristics, Diagnostic Evaluation, and Antibiotic Prescribing Patterns for Skin Infections in Nursing Homes . Front Med . 2016;3:30. https://www.frontiersin.org/ article/10.3389/fmed.2016.00030.

47. CDC. HAI and antibiotic use prevalence survey. Center for Disease prevention and Control (CDC). https://www.cdc.gov/hai/eip/antibiotic-use.html.

48. Eure T, Laplace LL, Melchreit R, et al. Measuring Antibiotic Appropriateness for Urinary Tract Infections in Nursing Home Residents. Infect Control Hosp Epidemiol 2017. doi:10.1017/ice.2017.96

49. Thompson ND, Penna A, Eure TR, et al. Epidemiology of Antibiotic Use for Urinary Tract Infection in Nursing Home Residents. J Am Med Dir Assoc. 2020;21(1):9196. doi:10.1016/j.jamda.2019.11.009
50. Daneman N, Gruneir A, Newman A, et al. Antibiotic use in long-term care facilities. J Antimicrob Chemother. 2011. doi:10.1093/jac/dkr395

51. Smith PW, Nicolle LE. The Chronic Indwelling Catheter and Urinary Infection in Long-Term-Care Facility Residents. Infect Control Hosp Epidemiol. 2001. doi:10.1086/501908

52. Vega RA, Conde JG, Díaz M. Prevalence of tuberculin reactivity and prevalence of risk factors for the development of active tuberculosis in a nursing home in Puerto Rico. P R Health Sci J. 1996.

53. Fu CJ, Mantell E, Stone PW, Agarwal M. Characteristics of nursing homes with comprehensive antibiotic stewardship programs: Results of a national survey. Am J Infect Control. 2020;48(1):13-18. doi:10.1016/j.ajic.2019.07.015

54. CDC. The core elements of antibiotic stewardship for nursing homes. Center for Disease prevention and Control (CDC). https://www.cdc.gov/longtermcare/ prevention/antibiotic-stewardship.html. Published 2020.

55. Metlay JP, Waterer GW, Long AC, et al. Diagnosis and treatment of adults with community-acquired pneumonia. An official clinical practice guideline of the American Thoracic Society and Infectious Diseases Society of America. Am J Respir Crit Care Med. 2019;200(7):E45-E67. doi:10.1164/rccm.201908-1581st

56. Sloane PD, Tandan M, Zimmerman S. Preventive antibiotic use in nursing homes: A not uncommon reason for antibiotic overprescribing. Journal of the American Medical Directors Association. 2020 Sep 1;21(9):1181-5.

57. Sloane PD, Zimmerman S, Ward K, et al. A 2-Year Pragmatic Trial of Antibiotic Stewardship in 27 Community Nursing Homes. J Am Geriatr Soc. 2020 doi: $10.1111 /$ jgs.16059 